\title{
FACTORES ENDÓGENOS Y EXÓGENOS ASOCIADOS AL RENDIMIENTO EN MATEMÁTICA: UN ANÁLISIS MULTINIVEL
}

\author{
Tania Elena Moreira Mora \\ Asesora Psicoeducativa del Departamento de \\ Orientación y Psicología \\ Instituto Tecnológico de Costa Rica \\ Cartago, Costa Rica
}

Recibido 3-III-2009 • Aceptado 9-IX-2009 • Corregido 6-X-2009

\begin{abstract}
Resumen: El propósito de este estudio fue identificar las variables exógenas y endógenas asociadas al rendimiento en la prueba de Matemática de Bachillerato en Educación Media, aplicada a los estudiantes de colegios públicos académicos diurnos de la modalidad formal del sistema educativo de Costa Rica, en la convocatoria ordinaria 2004. De acuerdo con los resultados estadísticos del modelo multinivel, se comprobó tanto la asociación de algunas variables exógenas de la institución, del grupo docente y su relación con el estudiantado, así como el historial académico y el contexto familiar en el nivel de los factores endógenos. Este estudio debe considerarse como una aproximación a un fenómeno educativo sumamente complejo como lo es el rendimiento en Matemática.
\end{abstract}

Palabras clave: factores, multinivel, rendimiento $y$ matemática.

\section{Introducción}

El área temática de esta investigación concierne a los factores asociados al rendimiento en la prueba nacional de bachillerato en Matemática del sistema educativo formal de Costa Rica, aplicada en la convocatoria ordinaria de 2004. Con este estudio se intenta establecer si existe o no una asociación de los factores endógenos y exógenos con respecto a las puntuaciones obtenidas por el estudiantado de undécimo proveniente de colegios públicos académicos diurnos en la prueba nacional de bachillerato.

En esta área investigativa existe una variedad de modelos e indicadores que han permitido mostrar diferentes percepciones y propósitos, unidades de análisis (micro, meso y macro nivel), alcance de modelos (primaria, secundaria o superior) y aproximaciones (input-output, proceso, organizativa y evaluativa entre otras); no obstante, en una revisión de la bibliografía, los modelos conceptuales coinciden en un conjunto de indicadores agrupados en cuatro grandes factores: contexto, input, 


\begin{abstract}
The purpose of this study was to identify the exogenous and endogenous variables associated to the students' academic performance in the Mathematics Secondary Education Test administered to students in Costa Rican public school during 2004. The statistical results of the multilevel model validated the association of some variables that are exogenous to the institution, to the faculty and its relation with the students; as well as the academic historical records and the family context at the endogenous level factors. This study must be considered as an approach to an extremely complex educational phenomenon such as the academic performance in Mathematics.
\end{abstract}

Key words: Factors, multilevel, achievement and mathematics. proceso y producto, aunque varían en su centro de atención y amplitud (Fernández y González, 1997). Estos modelos integradores, según los autores, suponen un avance importante no sólo en la definición teórica de los modelos, sino también en el mejoramiento técnico del análisis de datos.

En este estudio interesan algunos factores exógenos referentes a las relaciones socio-dinámicas, las estrategias metodológicas implementadas en los salones de clases y las características del cuerpo docente y de las instituciones educativas. En cuanto a los factores endógenos del estudiantado se incluyeron el perfil demográfico, las condiciones familiares, los antecedentes académicos y la disposición hacia la Matemática.

La razón práctica para enfocar la investigación hacia los factores asociados al rendimiento en la prueba de Matemática deriva de la comparación entre los resultados de las pruebas nacionales de bachillerato en Matemática, Español, Estudios Sociales, Idiomas Extranjeros y Ciencias, donde se evidenció que Matemática ha sido la asignatura con los más bajos porcentajes de promoción; por ejemplo, según información proporcionada por el Departamento de Pruebas Nacionales de la Dirección de Gestión y Evaluación de la Calidad del Ministerio de Educación Pública, en el lustro del año 2000 al 2004 en bachillerato aprobó, en promedio, solamente el 53\% de los estudiantes.

Por lo demás, la Matemática es una asignatura de suma relevancia en el currículo nacional y en el proceso educativo por sus aportes a otras ramas de la ciencia y por el desarrollo del pensamiento lógico y sistemático. Como lo destaca Azcárate (1998), su inclusión en el currículo de la enseñanza obligatoria se justifica por considerar a la Matemática como una parte fundamental del conocimiento que todo ciudadano debe poseer como bagaje cultural mínimo. Por ello, es una necesidad profundizar en los factores asociados a los bajos porcentajes de aprobación en 
esta prueba que, indiscutiblemente, reflejan deficiencias tanto en el proceso de enseñanza, responsabilidad del personal docente, como en el proceso de aprendizaje de los estudiantes. La identificación de tales factores contribuiría a la toma de decisiones pedagógicas e institucionales, tendientes a mejorar el rendimiento en esta prueba. La investigación de la cual se da cuenta en este artículo fue desarrollada en la Dirección de Gestión y Evaluación de la Calidad del Ministerio de Educación Pública.

\section{Pregunta de investigación}

¿Cuáles son los factores endógenos y exógenos asociados al rendimiento académico en la prueba nacional de bachillerato de Matemática de los estudiantes provenientes de colegios académicos públicos diurnos en el 2004?

\section{Objetivo general}

Establecer si existe o no relación entre los factores endógenos y exógenos con el rendimiento académico en la prueba nacional de bachillerato de Matemática de los estudiantes provenientes de colegios académicos públicos diurnos en el 2004.

\section{Marco referencial}

El rendimiento académico ha sido un tema de alto interés educativo e investigativo, analizado desde diversas perspectivas paradigmáticas y contextos. No obstante, este es un constructo sumamente complejo en el que intervienen condiciones endógenas y exógenas del estudiantado, cuyas relaciones no son fáciles de esclarecer, como es reconocido en múltiples investigaciones realizadas en América Latina en las dos últimas décadas del siglo XX (Vélez, Schiefelbein y Valenzuela, 1993). De acuerdo con lo que manifiesta Martínez
(2002), el rendimiento académico depende, en mayor o menor grado, de numerosas variables que configuran una enmarañada red en la que es harto complejo calibrar la incidencia específica de cada una.

Los factores endógenos analizados en esta investigación son el perfil demográfico, las condiciones familiares, los antecedentes académicos y la disposición hacia la Matemática del estudiante de undécimo. En tanto que los factores exógenos conciernen a las características del docente, estrategias metodológicas, relaciones socio-dinámicas y al escenario institucional. La selección de estos factores se ha sustentado en los hallazgos encontrados en múltiples investigaciones hechas en esta área temática como se describen en este apartado.

\section{Factores endógenos}

La motivación, la valoración o la disposición hacia la Matemática ha sido uno de los factores endógenos asociados al rendimiento escolar. Como lo destaca Cervini (2003), cuanto más intensas sean, mayor será el rendimiento. Asimismo, apunta que el aprendizaje dependerá de la perseverancia o esfuerzo del alumno que, a su vez, se relaciona con su motivación, concebida como una mezcla de capital cultural heredado familiarmente y de experiencias escolares con el incentivo, que el docente adopta en los procesos de enseñanza y aprendizaje.

Indiscutiblemente, en relación con la perseverancia y el esfuerzo, se ha comprobado que también los hábitos de estudio tienen un gran poder predictivo en el rendimiento académico, mayor incluso que las aptitudes intelectuales (Martínez, 2002). Sin embargo, Andrade, Miranda y Freixas (2000) encontraron que el máximo de predicción del rendimiento en Matemática fue la inteligencia lógica-matemática con un $14,2 \%$; más la inteligencia lingüística que aporta un $1,9 \%$; las dos, en su conjunto, logran predecir el 16,1\% de la dispersión del rendimiento en esta asignatura. 
Asimismo, se ha encontrado que las actitudes y aptitudes del estudiantado influyen fuertemente sobre su rendimiento académico por el papel predisposicional de las primeras y el desarrollo de habilidades cognitivas en las segundas. Así, las actitudes negativas no generan aprendizajes erróneos, sino que provocan que los estudiantes inicien sus actividades educativas más tarde y con menor interés, lo que multiplica sus errores y disminuye la eficacia de su aprendizaje (Gairín, 1990; González, 1989).

Otro factor endógeno relacionado con el rendimiento académico ha sido el historial académico del estudiantado. En una investigación, Cervini (2003) encontró dos interacciones significativas: una referida a los antecedentes académicos del alumnado (repetición); la otra, a su origen social. En la primera se comprobó que las diferencias de rendimiento entre el alumnado no repetidor y repetidor disminuyen en escuelas con indicios de clima académico negativo y ese acortamiento se debe particularmente a la caída del rendimiento promedio de los no repetidores. La segunda, altamente significativa, mostró que en la escuela donde el promedio de valoración positiva del aprendizaje es alta, disminuye el efecto del origen social del grupo estudiantil (educación de los padres) sobre el rendimiento. Entonces, mientras la primera interacción alude a la mayor desigualdad (referida a la aptitud), la segunda identifica resultados de equidad social, demostrando la complejidad de las determinaciones en el sistema educativo.

En cuanto a la influencia del factor familiar en el desarrollo psicosocial y cultural del estudiantado, se ha encontrado que el tipo de estimulación recibida en el hogar entre el nacimiento y el comienzo de la escolaridad y durante los periodos de vacaciones, así como las actividades recreativas, son fundamentales para estimular sus habilidades cognoscitivas, su desarrollo emocional y social. Además, han coadyuvado a mejorar el rendimiento académico (Martínez, 2002; Mella y Ortiz, 1999). Asimismo, durante los años de escolaridad, el nivel educativo de los padres es esencial en el desempeño escolar de los estudiantes y en su capacidad para alcanzar niveles superiores de escolaridad. De igual manera, las variables contextuales en torno a la madre son poderosos factores explicativos en el rendimiento en Matemática y Castellano, especialmente las expectativas de la madre con respecto a la carrera educacional de su hijo (Gairín, 1990; Mella y Ortiz, 1999; Reimers, 2003).

También las explicaciones referentes al estatus económico, tales como el ingreso familiar y el lugar de residencia, destacan que las condiciones de vida en general, el tipo de actividades dominantes en la comunidad, los ejemplos y los incentivos que inciden sobre los estudiantes juegan un papel favorable o restrictivo en el tipo de trabajo intelectual valorado por la escuela (González, 1989; Mella y Ortiz, 1999; Reimers, 2003). No obstante, se ha encontrado que no es el capital económico de la familia del alumno o del contexto institucional el de mayor relevancia para dimensionar la injusticia en la distribución de los aprendizajes, sino el capital cultural familiar y contextual el que moldea el perfil de la distribución del logro escolar (Cervini, 2002).

En otro estudio se confirma la relación entre la estructura familiar y el rendimiento de los alumnos de octavo grado en Matemática y lectura (Pong, 1997), al encontrar evidencias que sugieren que los niños procedentes de familias de un solo padre se inclinan a mostrar un menor rendimiento en las pruebas estandarizadas y tienen menos probabilidades de concluir la secundaria o de ingresar a la universidad con respecto a los niños que viven con ambos padres. En concreto, los estudiantes de familias con dos padres biológicos obtuvieron cuatro puntos más en las prueba de Matemática y lectura que los estudiantes con un solo padre biológico. En suma, es la presencia y apoyo afectivo de los padres una clave en el desarrollo integral y de destrezas relacionadas con el desempeño académico de los hijos. 
En definitiva, el desempeño académico resulta ser una variable muy compleja y multidimensional, pues en él se conjugan variables endógenas como la actitud, el esfuerzo, la motivación, las expectativas de éxito y las habilidades cognitivas; así como algunos factores familiares, académicos y características demográficas del estudiantado entre otros.

\section{Factores exógenos}

Uno de los factores exógenos más estudiados en el rendimiento académico ha sido el rol pedagógico de los docentes. En este sentido, Cervini (2003) cita el estudio reciente conducido por Yasumoto, Vekawa y Bidwell sobre el efecto de la cultura pedagógica de profesores de Matemática y Ciencias de secundaria en el rendimiento. Uno de sus hallazgos es que los docentes buscan resolver problemas endémicos del aula por medio de las interrelaciones informales cotidianas con sus colegas. Tales nudos de interacción generan una cultura local y una convergencia sobre la forma efectiva de enseñar y manejar el ambiente de aula, lo que produce una mayor consistencia colectiva en la práctica pedagógica de los docentes y, en consecuencia, intensifica el efecto sobre el progreso en el logro del alumnado.

En relación con el docente, en una revisión de 18 informes de investigación que incluían modelos de estimación o ecuaciones de regresión realizadas en América Latina en los últimos 20 años del siglo XX, Vélez et al. (1993) encontraron que la mayoría estaban enfocadas en la formación y experiencia docente, entre cuyos resultados se destacan que la experiencia docente (25 de 62 modelos) y el nivel académico de los docentes (31 de 68 modelos) están asociados significativamente con el rendimiento académico. Por otra parte, según los autores, la capacitación de los docentes en servicio no mejora el rendimiento de los estudiantes (7 de 8 modelos no muestra asociación), aunque desafortunadamente los modelos revisados no incluían los indicadores de la calidad de la capacitación y/o actualización, sólo hacían referencia a la experiencia de capacitación.

Otro factor exógeno asociado al rendimiento académico han sido las relaciones sociales o intersubjetivas del estudiantado. Desde la perspectiva de Andrade et al. (2000), los adolescentes asumen una subjetividad para interpretar el mundo y para entenderse a sí mismos; por ello las demás personas tienen una importancia especial para los jóvenes y las propias apreciaciones y valoraciones sobre sí mismos cobran nuevas dimensiones que los proyectan positiva o negativamente ante el mundo y sus tareas, particularmente en lo que respecta al rendimiento académico. Inclusive las relaciones entre los propios alumnos desempeñan un importante papel en el proceso de socialización.

Por otra parte, el efecto de las instituciones educativas en el estudiantado ha sido un factor endógeno estudiado con amplitud. De acuerdo con Mella y Ortiz (1999), hay una acumulación importante de conocimientos acerca de los factores institucionales y de la práctica pedagógica en el aula, que se presume inciden en el comportamiento escolar del alumno; de allí que las investigaciones han puesto un énfasis mayor en el estudio de los factores escolares, de los impactos de las variables internas del proceso de enseñanza y aprendizaje y del control metodológico adecuado de las relaciones entre factores escolares y extraescolares; por lo tanto, se puede presumir que el sistema escolar debería tener cierta capacidad de intervención y contrarrestar, en alguna medida, el impacto de las condiciones socioculturales de los alumnos.

Precisamente en un estudio realizado sobre el rendimiento de los alumnos del octavo grado en Matemática y lectura, cuyos padres fueron entrevistados en el estudio NELS 88 (National Education Longitudinal Study of 1988), provenientes de 970 escuelas de Estados Unidos, se encontró evidencias de que las escuelas rurales tienden a tener estudiantes con mejor rendimiento que las 
urbanas. Los resultados de la matrícula escolar y de la localización geográfica son consistentes con la explicación del capital social; puesto que en los centros más pequeños y en comunidades rurales se observan relaciones sociales más estrechas, redes más fuertes, todo lo que genera un capital social más grande y mejora la educación del niño (Pong, 1997).

En otra investigación, al relacionar la composición estudiantil de la escuela y algunas características del proceso escolar, según percepciones del alumno, con el rendimiento académico en Matemática de los estudiantes del último año de secundaria en Argentina, se encontró que las variables personales del alumno afectan principalmente la variación inter-escuela y no la variación intra-escuela; lo que puede interpretarse como una mayor homogeneidad social, ya sea por un alto nivel de selectividad o por una segmentación social de la red institucional del sistema educativo. Asimismo, se evidenció estadísticamente que el capital cultural familiar y contextual es el que moldea el perfil de la distribución del logro escolar y no el capital económico familiar y, finalmente, el efecto de la composición escolar sobre el rendimiento es mayor que el atribuible al conjunto de indicadores de antecedentes familiares y personales del alumnado. Es más, resultó altamente significativo que en las escuelas con un alto promedio de valoración positiva del aprendizaje escolar (expectativa de éxito futuro) disminuye el efecto del origen social del estudiante sobre el rendimiento (Cervini, 2002; 2003).

También la buena enseñanza depende del entorno físico donde se estudia, pues la iluminación, la temperatura, la ventilación, el ruido o el silencio, así como el mobiliario, son algunas condiciones que influyen en el estado del organismo y, por ende, en la concentración del estudiante. En este tópico, Vélez et al. (1993) encontraron que la mayoría de los indicadores de infraestructura considerados en varias investigaciones latinoamericanas, incluyendo no solo la calidad de la construcción sino también mobiliario y servicios de electricidad y agua, presentan un efecto positivo en el rendimiento (23 de un total de 70 modelos estadísticos), mas en un número considerable (45 de 70) no hubo ninguna asociación. También hay evidencias de un mejor rendimiento académico en los alumnos que trabajan en un ambiente presidido por normas claras, donde prevalezca la cooperación y las relaciones personales positivas, más que la competitividad (Martínez, 2002).

En síntesis, se puede afirmar que la institución educativa ejerce efectos positivos o negativos durante el proceso formativo de los estudiantes y, consecuentemente, en el rendimiento académico. Además, se reconoce el peso de la cultura institucional, el capital social, las relaciones intersubjetivas y las condiciones de infraestructura en su desempeño académico.

Por último, el rendimiento académico es un campo que presenta múltiples problemas que deben afrontarse. Algunos se vinculan con los procesos de medición, que normalmente se fundamentan en dos tipos de estrategias, por un lado las calificaciones escolares y por el otro, las pruebas objetivas, sean de aula o estandarizadas (Matas, 2003). En este estudio, el rendimiento académico se define como la puntuación obtenida por los estudiantes en la prueba nacional de Matemática al concluir la Educación Diversificada del sistema educativo formal. Obviamente, esta concepción limita un constructo sumamente complejo, que se puede explorar desde diversas dimensiones cognoscitivas y aptitudinales, no obstante, para efectos de esta investigación se fundamenta en el resultado empírico de una prueba.

\section{Marco metodológico}

Este es un estudio correlacional predictivo; esto es, según Colás y Buendía (1994), un tipo de investigación que trata de 
conocer el efecto y magnitud de determinadas variables en la predicción de un determinado fenómeno, usualmente apoyado en modelos correlacionales y de regresión. En este análisis, la variable dependiente es la puntuación de la prueba de bachillerato, en tanto que las independientes se clasificaron en dos conglomerados: el primero es "endógenas", constituido por las variables demográficas, familiares, historial académico y disposición hacia la Matemática de los estudiantes; y el segundo, las variables "exógenas", conformado por las estrategias metodológicas, relaciones socio-dinámicas, características del docente y de la institución. Por la imbricación y complejidad de estas variables, el procedimiento estadístico multinivel es el más adecuado para determinar las relaciones con respecto a la puntuación de la prueba de Matemática.

Este modelo multinivel es utilizado con frecuencia en estudios del área de la educación, debido la complejidad del sistema educativo, a la naturaleza jerárquica o de conglomerados de la organización escolar -estudiantes, grupos e institución- y al impacto potencial de esos niveles en las puntuaciones de los examinados (Crislip y Heck, 2001; Hwang, 2002; Paterson, 1998; Young, 1997). Para ampliar la descripción y ventajas de este procedimiento multinivel se puede recurrir a Malin y Linnakyla (2001) y a Kreft (1990).

El análisis estadístico se realizó con el programa Statistical Package for Social Sciences (SPSS), en su versión 12,0 para el entorno Windows, y se empataron los datos de los estudiantes con las observaciones de sus respectivos docentes. Posteriormente, se aplicó el modelo de estimación o ecuaciones de regresión múltiple con dos niveles. De acuerdo con Murillo (1999), con este análisis se muestra la relación entre las variables dentro de un determinado nivel y se especifica cómo las variables de ese nivel influyen en las relaciones establecidas en otros niveles. Para realizar este análisis multinivel se utilizó el paquete estadístico STATA, versión 4,0.

\section{Hipótesis}

En esta investigación interesa identificar los factores endógenos y exógenos asociados al rendimiento en las pruebas nacionales de Matemática, por lo tanto, se asumen las siguientes hipótesis.

Primera hipótesis: El rendimiento académico en la prueba nacional de bachillerato de Matemática de los estudiantes procedentes de colegios académicos públicos diurnos está relacionado significativamente con características del espacio institucional.

Segunda hipótesis: El rendimiento académico en la prueba nacional de bachillerato de Matemática de los estudiantes procedentes de colegios académicos públicos diurnos se asocia significativamente con las relaciones socio-dinámicas, estrategias metodológicas y características del docente.

Tercera hipótesis: El rendimiento académico en las pruebas nacionales de Matemática de noveno y undécimo año de colegios académicos públicos diurnos se relaciona significativamente con algunas variables demográficas, familiares, historial académico y con la disposición hacia la Matemática del estudiante.

\section{Muestra}

El criterio para seleccionar al estudiantado de undécimo año fue su condición académica, ya que al concluir la Educación Diversificada deben realizar las pruebas nacionales de certificación. La población nacional de undécimo año fue de 17.764 estudiantes provenientes de 204 colegios públicos académicos diurnos. Esta información fue proporcionada por el Departamento de Análisis Estadísticos del Ministerio de Educación Pública de Costa Rica, con base en la matrícula inicial del 2004. De esta población se excluyeron 
los estudiantes de las telesecundarias, las unidades pedagógicas y los programas no tradicionales del sistema educativo.

El tamaño de la muestra fue de 1.468 estudiantes y se estableció de acuerdo con los siguientes parámetros: un nivel de confianza del 95,5\%, un margen de error permitido de $2,5 \%$ y un estimado de la proporción de la población igual a 0,50 (variancia). No obstante, durante la administración de las encuestas no se encontraron algunos de los estudiantes seleccionados aleatoriamente, ya sea porque ese día no se presentaron a la institución o se habían retirado, entre otras circunstancias; debido a esto, la muestra final quedó conformada por 1.364 estudiantes.

La muestra fue probabilística, pues cada uno de los 204 colegios públicos académicos diurnos de Costa Rica que impartían undécimo año tuvieron la misma posibilidad de ser seleccionados. Específicamente, fue una muestra estratificada entre las veinte direcciones regionales de enseñanza del Ministerio de Educación Pública. Con respecto a los docentes de Matemática, el muestreo fue no probabilístico, ya que debían seleccionarse aquellos que impartían lecciones a los estudiantes de undécimo de los 36 colegios muestreados.

\section{Instrumentos}

El instrumento aplicado a los docentes de Matemática de los colegios seleccionados se estructuró en dos partes. La primera medía algunas características generales con ítems de selección única y la segunda estaba conformada por distintos diferenciales semánticos y escalas de tipo Likert para medir sus percepciones sobre múltiples dimensiones pedagógicas e institucionales.

El instrumento administrado al estudiantado de undécimo año también se estructuró en secciones. La primera referida a las características demográficas medidas con ítems de selección. La segunda valoró la disposición hacia la Matemática con ítems de selección y una escala de tipo Likert. En la última sección se incorporaron las condiciones académicas del estudiantado, las que se midieron con ítems de selección, de respuesta abierta y tres escalas de tipo Likert. En total, el instrumento quedó constituido por un total de 97 reactivos, de los cuales 27 se tomaron de una encuesta elaborada por Rojas (2004). La recolección de datos se realizó antes de la aplicación de la prueba nacional de Matemática, en un periodo de dos meses, comprendido entre 28 de julio y el 27 de setiembre del 2004, con la colaboración de funcionarios de la Dirección de Gestión y Evaluación de la Calidad.

El procedimiento para confeccionar estos instrumentos se realizó siguiendo los siguientes pasos:

1. Análisis documental de diversas publicaciones concernientes a los factores asociados al rendimiento académico, con énfasis en Matemática, tanto para definir el marco referencial como para revisar los instrumentos utilizados en investigaciones similares.

2. Redacción de los ítems para medir las variables en estudio.

3. Construcción de una versión preliminar del instrumento.

4. Validación de cada instrumento con el criterio de cinco expertos.

Además, en el caso de los estudiantes, se realizó una prueba piloto el 15 de abril del 2004 en un colegio público académico diurno de la Dirección Regional de Puriscal. Se seleccionaron cuarenta estudiantes de undécimo ubicados en tres grupos diferentes, con un total de dieciséis mujeres y veinticuatro varones.

En cuanto a la calidad técnica del instrumento de los estudiantes se obtuvieron, por una parte, evidencias asociadas a la validez de contenido mediante la opinión de jueces. Por la otra, se estimó la 
confiabilidad de las escalas tipo Likert, que medían: la disposición hacia la Matemática (13 ítems), la relación con el docente (9 ítems), la percepción sobre la metodología (12 ítems) y el uso de los recursos didácticos en las lecciones de Matemática (9 ítems). En este estudio se utilizó el coeficiente alfa de Cronbach, especificado como una evidencia de la exactitud de las medidas. Según Kerlinger (1988), la consistencia interna de una prueba significa que las características de la prueba son homogéneas. En concreto, el grado de consistencia de las puntuaciones en las escalas fue de 0,93 , suficientemente aceptable para efectos de investigación, como lo destacan Nunnally y Bernstein (1995).

\section{Definición de las variables}

La construcción operativa de los instrumentos se fundamentó en la definición sustantiva de las variables exógenas y endógenas incluidas en el modelo multinivel de dos conglomerados, las cuales se asociaron con el rendimiento académico en Matemática, variable dependiente.

\section{Definiciones sustantivas}

Definiciones operativas

\section{Demográficas}

La clasificación por edades y sexos es la estructura poblacional más utilizada puesto que gran parte del comportamiento demográfico está condicionado por estas dos dimensiones (Ortega, 2001).

\section{Familiares}

La familia es un grupo socialmente construido, heterogéneo, diverso, dinámico e histórico, que mediante lazos de parentesco establece un tipo de relación particular (Franco y Tobasura, 2007).

\section{Historial académico}

Cuando los estudiantes tienen confianza en su capacidad de éxito, aceptan los desafíos planteados en nuevas tareas y persisten en su esfuerzo para realizarlas con éxito, mientras que al experimentar decepciones y dudas de su capacidad los cwonducen a alcanzar resultados negativos (García y Doménech, 1997).

\section{Disposición}

Es la conjunción de percepciones, sentimientos, creencias, intenciones, metas y formas de pensar y valorar que van condicionando la capacidad para aprender, la realización de tareas, la autodeterminación para enfrentar los retos, el trabajo en equipo, la curiosidad e interés por conocer y la confianza en las propias capacidades (García y Doménech, 1997).
Los valores absolutos y porcentuales se obtuvieron de una encuesta aplicada a estudiantes y a docentes sobre el sexo, la edad, la nacionalidad y el tener hijos.

Los valores absolutos y porcentuales obtenidos de una encuesta aplicada a los estudiantes acerca del núcleo familiar, nivel de escolaridad de los padres y madres, el total de integrantes de la familia, su ingreso mensual, el financiamiento de los estudios, estado de la casa, sus bienes y acceso a los servicios.

Los valores absolutos y porcentuales se obtuvieron de una encuesta aplicada a estudiantes sobre los antecedentes académicos de cada estudiante en primaria y secundaria, cumplimiento de sus deberes escolares y tiempo de estudio, así como el nivel académico, profesional y la universidad de procedencia de los docentes.

Los valores absolutos y porcentuales se obtuvieron de una encuesta aplicada a estudiantes acerca de la disposición de los estudiantes para aprender Matemática, sus creencias y el apoyo para estudiar y cumplir con sus deberes académicos. 


\begin{abstract}
Definiciones sustantivas
Estrategias metodológicas

La enseñanza promueve la independencia y la responsabilidad del alumno y un mayor desarrollo de la metacognición, bajo el principio metodológico de la flexibilidad que permita un constante intercambio, realimentación y reajuste del currículo; es una enseñanza más ajustada a las necesidades personales del educando y a sus características (Sainz, 1998).
\end{abstract}

\section{Relaciones socio-dinámicas}

Es la relación entre docente y estudiantes, sustentada en el reconocimiento, por parte del primero, de la incidencia directa de las formas de comunicación e interacción en los procesos de aprendizaje y de la importancia de valorar y prestar atención a la dimensión subjetiva de ambos (Ordoñez, 2006).

\section{Institucional}

Es el marco de referencia de los actores participantes que realizan actividades intencionalmente educativas en escenarios particulares, el cual permite comprender e interpretar las diversas formas de intercambio en torno al conocimiento matemático (Gutiérrez, 1994).

\section{Rendimiento académico}

Es el producto dado por el estudiante en los centros de enseñanza y que habitualmente se expresa mediante las calificaciones (Martínez, 1996).

\section{Definiciones operativas}

Los valores absolutos y porcentuales se obtuvieron de una encuesta aplicada a estudiantes y docentes sobre los procedimientos y recursos didácticos utilizados por el docente para enseñar Matemática en el aula.

Los valores absolutos y porcentuales obtenidos de la encuesta aplicada a estudiantes y a docentes sobre las interacciones dentro del ámbito institucional y regional.

Los valores absolutos y porcentuales se obtuvieron de la encuesta aplicada a estudiantes sobre las condiciones físicas, ubicación geográfica, servicios del colegio, tiempo de traslado y medios de transporte.

Es la puntuación obtenida por los estudiantes en la prueba nacional de bachillerato en Matemática al concluir el colegio.

\section{Análisis de los datos}

El análisis inferencial se basa en los resultados del modelo multinivel, donde se empataron algunas variables del estudiante, identificadas con la abreviatura "et", con los datos del respectivo docente de Matemática, reconocidas con la abreviatura "pf". Estas variables se recodificaron con valores 0 y 1 y se asociaron con la correspondiente puntuación obtenida por el estudiante en la prueba nacional de Matemática de bachillerato. Además de esta ventaja, en este modelo se logra controlar el efecto de las otras variables independientes, y por lo tanto es un análisis estadísticamente más poderoso.

Este análisis se basó en el modelo de regresión lineal con variables independientes a dos niveles de agregación, en el cual se perdió un número considerable de observaciones debido a los datos faltantes tanto del estudiante como del docente, ya que en el modelo de regresión se incluyen únicamente los casos con datos completos; por ello, el análisis se ejecutó con un total de 482 observaciones y no se utilizó ninguna técnica para completar los datos faltantes. Asimismo, uno de los propósitos de este análisis es determinar cuánta variancia es explicada por las variables incluidas en el modelo. De acuerdo con el coeficiente de determinación, la variancia explicada por las variables independientes incluidas en el análisis fue de $30 \%$; lo que significa que el $70 \%$ restante se relaciona con otros factores no incorporados en el modelo multinivel. 
Asimismo, cabe agregar que antes de incluir las variables en el modelo de regresión, se realizó el análisis de multicolinealidad para eliminar las variables independientes con correlaciones altas, puesto que cuanto más variables se incorporen dentro de la ecuación, más posibilidades hay de que exista colinealidad. El indicador asumido en esta evaluación fue el valor de tolerancia (próximo a 0,01); según Pardo y Ruiz (2002), significa que es una variable que comparte el $99 \%$ de la variancia con las restantes independientes; por lo tanto, se trata de una variable redundante. En la ecuación de este modelo de regresión se excluyeron tres variables por presentar problemas de colinealidad, según el indicador de tolerancia. Entonces se incorporaron un total de 12 variables independientes del docente y 25 del estudiante.

$\mathrm{Al}$ asumirse en esta investigación que el constructo medido, rendimiento en la prueba de Matemática, se asocia con múltiples variables interrelacionadas, se consideró un nivel de significación estadística igual o inferior a 0,1 $(\mathrm{P}>\mathrm{t})$. De acuerdo con las estimaciones, si una variable no es significativa, entonces el coeficiente de regresión es cero, es decir, no existe ninguna relación, pero al resultar con significancia estadística sí aporta una explicación a la variación en el rendimiento de la prueba. Además, implica que los resultados obtenidos de esta muestra son válidos y extensibles estadísticamente a la población de examinados procedentes de colegios públicos, académicos y diurnos.

Tabla 1

Coeficientes de regresión del modelo multinivel de la prueba de bachillerato de Matemática aplicada en colegios académicos diurnos públicos en el 2004

\begin{tabular}{|c|c|c|c|c|c|}
\hline Variables & $\begin{array}{c}\text { Coeficiente } \\
\text { de } \\
\text { regresión }\end{array}$ & $\mathrm{P}>\mathrm{t}$ & Variables & $\begin{array}{c}\text { Coeficiente } \\
\text { de } \\
\text { regresión }\end{array}$ & $\mathrm{P}>\mathrm{t}$ \\
\hline $\begin{array}{l}\text { et_repitió algún nivel de la } \\
\text { escuela }\end{array}$ & $-4,314282$ & 0,210 & et_recursos didácticos & 5641675 & 0,600 \\
\hline et_edad del estudiante &,- 0477075 & 0,966 & et_trabajos extra clase &,- 842511 & 0,399 \\
\hline et_núcleo familiar & 1,315285 & 0,260 & et_contento en el colegio & 2,916403 & 0,290 \\
\hline et_escolaridad del padre & 0,9309091 & 0,524 & et_repitió en algún nivel & $-2,732938$ & 0,072 \\
\hline et_escolaridad de madre & $-5,058615$ & 0,001 & et_aplazó en algún nivel & $-4,863068$ & 0,000 \\
\hline et_comportamiento del padre & $-1,6163$ & 0,215 & et_estudió colegio privado & 1,878423 & 0,607 \\
\hline et_comportamiento de madre &,- 4359444 & 0,739 & pf_sexo del profesor & $-3,16879$ & 0,110 \\
\hline et_integrantes de hogar & 1,584598 & 0,126 & pf_edad del profesor & 4,4174 & 0,213 \\
\hline et_ingreso mensual del hogar & 0,2605557 & 0,825 & $\begin{array}{l}\text { pf_universidad de } \\
\text { procedencia }\end{array}$ & $-3,451026$ & 0,233 \\
\hline $\begin{array}{l}\text { et_ubicación geográfica del } \\
\text { colegio }\end{array}$ & $-2,015836$ & 0,138 & $\begin{array}{l}\text { pf_años de servicio del } \\
\text { docente }\end{array}$ & 3,882261 & 0,079 \\
\hline et_tiempo de traslado al colegio & 1,125707 & 0,293 & $\begin{array}{l}\text { pf_años servicio en } \\
\text { institución }\end{array}$ & ,4070573 & 0,859 \\
\hline et_transporte al colegio & 2,662763 & 0,022 & pf_grado académico & $-1,569344$ & 0,303 \\
\hline
\end{tabular}




\begin{tabular}{|c|c|c|c|c|c|}
\hline Variables & $\begin{array}{c}\text { Coeficiente } \\
\text { de } \\
\text { regresión }\end{array}$ & $\mathrm{P}>\mathrm{t}$ & Variables & $\begin{array}{l}\text { Coeficiente } \\
\text { de } \\
\text { regresión }\end{array}$ & $\mathrm{P}>\mathrm{t}$ \\
\hline et_horas de estudio por semana & 1,476001 & 0,242 & pf_condición laboral & -3.572302 & 0,055 \\
\hline et_estudió en escuela privada & $-2,771$ & 0,257 & pf_asesoramientos & $-3,421807$ & 0,102 \\
\hline et_actitud hacia la Matemática &,- 114629 & 0,919 & $\begin{array}{l}\text { pf_coordinación con el } \\
\text { director }\end{array}$ & 1,010882 & 0,697 \\
\hline et_bienes no básicos & 1,377175 & 0,236 & pf_relación con padres &,- 6006785 & 0,695 \\
\hline et_disposición hacia la Matemática &, 1515364 & 0,193 & pf_recursos didácticos & 3,193487 & 0,507 \\
\hline et_relación con el docente & 2,08694 & 0,138 & $\begin{array}{l}\text { pf_relación con los } \\
\text { esudiantes }\end{array}$ & $-9,722346$ & 0,084 \\
\hline et_metodología empleada profesor &,- 1470038 & 0,898 & _constante & 80,41479 & 0,000 \\
\hline
\end{tabular}

Fuente: Elaboración propia.

Número de observaciones $=484$

Coeficiente de determinación $=0,30$

La variación en las puntuaciones de la prueba de Matemática se asocia significativamente con siete variables del modelo; específicamente, con los factores denominados historial académico y familiares correspondientes al componente endógeno; mientras que entre las exógenas se identificaron ciertas variables relativas a las relaciones socio-dinámicas y a las características institucionales y del docente.

De las variables del historial académico de los estudiantes, incluidas en el modelo como se observa en la tabla 2 , dos aportan una explicación en la variación de las puntuaciones, la repetición y aplazamiento en algún nivel de la educación secundaria por la asignatura de Matemática.

Tabla 2

Porcentajes de las variables endógenas del historial académico de los estudiantes de undécimo en el 2004

\begin{tabular}{lcc}
\hline \multicolumn{1}{c}{ Variables } & Indicadores & Porcentaje \\
\hline Repetición de uno o más niveles en el colegio por Matemática. & No & 80,5 \\
& Sí & 19,5 \\
Aplazamiento en uno o más niveles en el colegio por Matemática. & No & 52,1 \\
& Sí & 47,9 \\
Estudió uno o más niveles en una escuela privada. & No Sí & 94,8 \\
& No & 5,2 \\
Estudió uno o más niveles en un colegio privado. & Sí & 94,9 \\
Cumple con los trabajos extraclase. & Todos & 5,1 \\
Horas de estudio por semana. & No todos & 68,8 \\
\end{tabular}

Fuente: Elaboración propia. 
Según los datos anteriores, hubo un alto porcentaje de aplazamiento en la educación secundaria por Matemática, pues de cada cien estudiantes, 48 se han quedado aplazados una o más veces; además, casi el $20 \%$ repitió algún nivel debido a la reprobación en Matemática. Dentro de este contexto, la interpretación del coeficiente del modelo de regresión es:

- Los estudiantes que aplazaron en uno o más niveles de la educación secundaria por Matemática tendieron a obtener, en promedio, 4,86 puntos menos en la prueba, manteniendo todos los demás factores constantes, en relación con los estudiantes que nunca aplazaron por esta asignatura.

- Los estudiantes que repitieron en uno o más niveles de la educación secundaria por Matemática tendieron a obtener, en promedio, 2,73 puntos menos en la prueba, manteniendo todos los demás factores constantes, en relación con los estudiantes que nunca aplazaron por esta asignatura.

La otra variable endógena con significancia estadística fue el nivel de escolaridad de la madre. De acuerdo con los datos de la tabla 3, el 66\% de los estudiantes vivían con ambos padres y predominaban las conductas afectivas por parte de ambos padres, principalmente de la madre. Quizás la condición económica de estos hogares constituya el principal riesgo de la estabilidad familiar, puesto que más del $50 \%$ tenían ingresos mensuales inferiores o iguales a US $\$ 300$, especialmente, para quienes vivían únicamente con la madre, el padre u otro familiar. Probablemente, estos bajos ingresos se relacionan con el nivel de escolaridad de ambos progenitores ya que, en promedio, únicamente el 74,25 \% realizó estudios primarios y secundarios, lo cual constituye una barrera para optar por puestos calificados y mejor remunerados.

Tabla 3

Porcentajes de las variables endógenas familiares de los estudiantes de undécimo en el 2004

\begin{tabular}{|c|c|c|}
\hline Variables & Indicadores & Porcentajes \\
\hline \multirow{2}{*}{ Núcleo familiar } & Vive con ambos padres & 66,4 \\
\hline & Otros familiares & 33,6 \\
\hline \multirow{2}{*}{ Escolaridad del padre } & Realizó estudios superiores & 28,6 \\
\hline & Realizó sólo estudios primarios y secundarios & 71,4 \\
\hline \multirow{2}{*}{ Escolaridad de la madre } & Realizó estudios superiores & 22,9 \\
\hline & Realizó sólo estudios primarios y secundarios & 77,1 \\
\hline \multirow{2}{*}{ Comportamiento del padre } & Conductas no afectivas & 20,6 \\
\hline & Conductas afectivas & 79,4 \\
\hline \multirow{2}{*}{ Comportamiento de la madre } & Conductas no afectivas & 8,6 \\
\hline & Conductas afectivas & 91,4 \\
\hline \multirow{2}{*}{ Total de integrantes de la familia } & De 3 a 5 miembros & 68,1 \\
\hline & De 6 o más miembros & 31,9 \\
\hline \multirow{2}{*}{ Ingreso mensual de la familia } & Menor o igual a $\phi 150000,00$ & 56,5 \\
\hline & Mayor a $\phi 150000,00$ & 43,5 \\
\hline
\end{tabular}

Fuente: Elaboración propia. 
Según el coeficiente de regresión, en promedio los examinados cuyas madres realizaron estudios superiores tendieron a obtener 5,06 puntos más en la prueba de Matemática, manteniendo todos las demás factores constantes, en relación con estudiantes que tenían madres con estudios primarios y secundarios únicamente.

En definitiva, en el análisis se evidenció que a mayor escolaridad de las madres de los estudiantes se tendió a obtener más puntos en la prueba de Matemática y los que aplazaron o repitieron algún nivel de la educación secundaria por la asignatura de Matemática tienden, en promedio, a obtener una menor puntuación en comparación con quienes nunca habían aplazado por esta asignatura.
En cuanto al historial académico de los docentes, el $75,2 \%$ tenía el grado de bachillerato $\mathrm{u}$ otro nivel superior de formación. Estos estudios los habían realizado principalmente en las universidades públicas $(84,2 \%)$ y algunos en privadas $(15,8 \%)$. En el contexto laboral, el $84,4 \%$ estaba en propiedad en el colegio actual (correspondiente a la muestra), de los cuales el 48,2\% tenía entre 1 y 4 años de laborar en la institución actual, y de 5 a 9 años, el 51,8\%. Estos datos de la tabla 4 marcan dos condiciones importantes, la estabilidad laboral y la experiencia en el colegio, las cuales podrían contribuir a un buen desempeño pedagógico y un mayor conocimiento de sus atributos profesionales y personales por parte del director, padres de familia y estudiantes.

Tabla 4

Porcentajes de las variables exógenas de los docentes de Matemática de undécimo en el 2004

\begin{tabular}{llc}
\hline \multicolumn{1}{c}{ Variables del docente } & Indicadores & Porcentajes \\
\hline Grado académico en Matemática & Grado inferior a bachillerato & 24,8 \\
& Bachiller y grados superiores & 75,2 \\
Universidad de procedencia & Privada & 15,8 \\
& Pública & 84,2 \\
Años de servicio como docente & De 1 a 9 años & 49,2 \\
& De 10 a más años & 50,8 \\
Años de servicio en la institución & & 48,2 \\
& De 1 a 4 años & 51,8 \\
Condición laboral & De 5 a 20 años & 84,4 \\
\hline
\end{tabular}

Fuente: Elaboración propia.

Dentro de las variables exógenas, mostraron una relación significativa con las puntuaciones en la prueba de Matemáticas los años de servicio como docente y la condición laboral, cuyos coeficientes de regresión se interpretan de la siguiente manera:
Los estudiantes instruidos por docentes con menos de 10 años de servicio, en promedio, obtuvieron 3,88 puntos más en la prueba, manteniendo todos los demás factores constantes, en comparación con los examinados preparados por docentes 
con más de 10 años de servicio como docentes de Matemática.

- Los estudiantes preparados por docentes interinos obtuvieron, en promedio, 3,6 puntos más en la prueba, manteniendo todos los demás factores constantes, en relación con los estudiantes con docentes en condición de propiedad.

La otra variable exógena cuyo coeficiente de regresión resultó significativo fue la relación de los docentes con los estudiantes, enfocada hacia la interacción del docente con el estudiante y viceversa, en el espacio de las aulas, durante el desarrollo de las lecciones de Matemática. Según los datos de la tabla 1, esto significa que:

- Los estudiantes cuyos docentes con frecuencia mantuvieron una relación comprensiva, prudente, tolerante, respetuosa, de confianza y diálogo, obtuvieron en promedio 9,72 puntos más en la prueba de Matemática, manteniendo todos los demás factores constantes, con respecto a aquellos estudiantes cuyos docentes casi nunca mostraban una relación positiva.

Finalmente, de las variables institucionales descritas en la tabla 5, se puede manifestar que aproximadamente el $60 \%$ de los estudiantes utilizó algún tipo de transporte (autobús, carro, bicicleta y otros) para llegar a su institución, ubicada relativamente cerca, pues el $77 \%$, en promedio, tardaba menos de 30 minutos, a pesar de que para el 35\% la ubicación geográfica era mala. No obstante, sobresalió un sentimiento positivo, pues el 93\% de los estudiantes manifestó sentirse contento de su condición de ser alumno de ese colegio en particular.

Tabla 5

Porcentajes de las variables exógenas de la institución, según los estudiantes de undécimo en el 2004

\begin{tabular}{llcc}
\hline & & \multicolumn{2}{c}{ Niveles } \\
\cline { 2 - 3 } Categorías & Indicadores & Noveno & Undécimo \\
\hline Ubicación geográfica del colegio. & Buena ubicación & 58,9 & 65,2 \\
& Mala ubicación & 41,9 & 34,8 \\
& & & 77,6 \\
Tiempo de traslado. & Menos de media hora & 24,2 & 22,4 \\
& Media hora o más. & & \\
Medio de transporte. & & 38,8 & 41,7 \\
& A pie & 61,2 & 58,3 \\
Se siente contento. & Utiliza algún medio de transporte & & 7,3 \\
& & & 92,7 \\
\hline
\end{tabular}

Fuente: Elaboración propia.

La única variable institucional que aporta un valor explicativo a la nota del examen de Matemática fue el tipo de transporte hacia la institución, cuya interpretación es:
En promedio, los estudiantes que utilizaban algún medio de transporte para llegar al colegio obtuvieron 2,66 puntos más en la prueba de Matemática, manteniendo todos 
los demás factores constantes, con respecto a quienes se trasladan caminando.

Finalmente, cabe aclarar que los resultados del modelo multinivel no son relaciones de causa-efecto, sino coeficientes que evidencian estadísticamente la relación entre las variables independientes con el rendimiento en la prueba de Matemática, cuyos resultados pueden ser afectados por algunas variables confusoras, a pesar de que este análisis es muy poderoso, pues controla el efecto de todas las variables incluidas dentro del modelo.

\section{Conclusiones}

Este estudio fue el resultado de una amplia recolección de información a nivel nacional y de un análisis integral con el propósito de determinar las variables exógenas y endógenas asociadas al rendimiento en la prueba de Bachillerato en Educación Media de Matemática aplicada en colegios públicos académicos diurnos de la modalidad formal del sistema educativo, en la convocatoria ordinaria aplicada en el mes de noviembre de 2004.

En relación con el propósito de esta investigación, se estableció la relación de algunas variables exógenas y endógenas con el rendimiento en la prueba de Matemática. Específicamente, en la primera hipótesis relativa a los factores exógenos del contexto institucional, la única variable con significancia estadística fue el tiempo de traslado. La segunda hipótesis concierne a los factores exógenos del docente, las variables denominadas condición laboral, años de servicio como docente y relación con los estudiantes; estos explican parte de la variación en las puntuaciones de la prueba de Matemática. En la tercera hipótesis, que incorpora los factores endógenos, resultaron significativos estadísticamente la escolaridad de la madre, la condición de repitente y de aplazamiento en algún nivel de la secundaria por Matemática.
A partir de estos resultados, se puede afirmar que estas siete variables agrupadas en dos niveles -endógeno y exógenoexplican un $30 \%$ de la variabilidad de las puntuaciones de la prueba de Bachillerato. Además, algunos de estos hallazgos son coincidentes con estudios de otros países latinoamericanos. Entre los factores endógenos, el historial académico del estudiantado, específicamente en la condición de repitencia (Cervini, 2003) y los años de escolaridad y el nivel educativo de los padres (Gairín, 1990; Mella y Ortiz, 1999; Reimers, 2003). En cuanto a las variables exógenas, la interacción del docente con sus estudiantes en el espacio del aula (Martínez, 2002) y algunas características de la formación y experiencia docente (Vélez et al., 1993). No obstante, cabe insistir que estos estudios son aproximaciones estadísticas a un fenómeno educativo muy complejo como es el rendimiento en una prueba nacional de Matemática, en el cual intervienen no solamente variables asociadas al estudiante y docente, sino también a otros agentes educativos como los administrativos, asesores de Matemática, personal de apoyo, padres de familia y miembros de la comunidad.

Por lo demás, estos resultados se pueden considerar un aporte para la toma de decisiones en el ámbito nacional, regional e institucional, pues uno de los propósitos es contribuir al mejoramiento de la práctica educativa y, consecuentemente, del rendimiento académico de los estudiantes de undécimo en la prueba nacional de Matemática. En el ámbito exógeno, se recomienda a los docentes propiciar un ambiente más abierto al diálogo y a la confianza, mediante la apertura de espacios reflexivos de manera que los estudiantes puedan expresar sus ideas y dudas. Así, con una mayor comunicación y comprensión entre estudiantes y docente, mejorarán indudablemente las relaciones sociodinámicas en el aula. También es importante considerar la estabilidad laboral y la experiencia como criterios para seleccionar el docente que imparte esta asignatura en el 
nivel de undécimo. Igualmente, es necesario brindar a los estudiantes algunos servicios de transportes para facilitar su traslado a la institución, puesto que esto contribuye a un mejor rendimiento académico.

En cuanto a los elementos endógenos, es preocupante la incidencia del historial académico en el rendimiento, puesto que aquellos estudiantes que repitieron o aplazaron algún grado se encuentran en una condición de desventaja con respeto a quienes han mostrado un dominio desde sétimo año. De ahí que una de las principales recomendaciones para los docentes y administrativos es darle un seguimiento a los estudiantes desde el primer año de secundaria para reforzar sus conocimientos y desarrollar destrezas básicas en Matemática, puesto que conforme avancen en los distintos grados de la educación secundaria el nivel cognitivo es más complejo, y por tanto, más difícil de superar sus limitaciones y deficiencias. Además, es necesario brindarles los apoyos académicos necesarios para fortalecer las metas de aprendizaje y la motivación, para que de alguna manera se reduzca el efecto del nivel educativo y del capital cultural de los padres y las madres de los estudiantes.

Algunas de las limitaciones de este estudio fueron tanto la ausencia de las opiniones de los padres, así como la profundización de la información mediante el empleo de técnicas cualitativas, lo que evidentemente se justifica por los pocos recursos humanos y económicos para implementarlas. Igualmente, la información faltante en las bases de datos de estudiantes y docentes fue otra restricción que repercutió en la disminución del tamaño de la muestra (472 en undécimo) en el modelo de regresión múltiple y que pudo afectar los resultados del análisis multinivel.

\section{Referencias bibliográficas}

Andrade, M., Miranda, C., y Freixas, I. (2000). Rendimiento académico y variables modificables en alumnos de 2do. medio de liceos municipales de la Comuna de Santiago. Consultado el 10 de noviembre del 2004, de: http://www.propedeutico.cl/data/ upload_pub/22958725676689441413. pdf

Azcárate, P. (1998). La investigación matemática. Cuestiones sobre los procesos de formación de los profesores. Revista Electrónica de Investigación y Evaluación Educativa, 3(2). Consultado el 14 de diciembre del 2005, de: http://www.uv.es/RELIEVE/v3n2/ RELIEVEv3n2_0.htm

Cervini, R. (2002). Desigualdades socioculturales en el aprendizaje de matemática y lengua de la educación secundaria en Argentina: Un modelo de tres niveles. Revista Electrónica de Investigacióny Evaluación Educativa, 8(2). Consultado 15 de diciembre del 2004, de: http://www.uv.es/ RELIEVE/v8n2/RELIEVEv8n2_1. htm

Cervini, R. (2003). Relaciones entre composición escolar, proceso escolar y el logro en matemática en la educación secundaria en Argentina. Revista Electrónica de Investigación y Evaluación Educativa, 5(1). Consultado el 16 de febrero del 2005, de: http://redie.ens.uabc.mx/vol5no1/ contenido-cervini2.html

Colás, M. P., y Buendía, L. (1994). Investigación educativa. España: Ediciones Alfar Universidad.

Crislip, M. A., y Heck, R. H. (2001). Accountability, writing assessment, and equity: Testing a multilevel model [Rendición de cuentas, evaluación de escritura y equidad: Prueba de un modelo multinivel]. Hawaii Department of Education 
y University of Hawaii, Manoa. Artículo presentado en la Reunión Anual de la Asociación Americana de Investigación Educativa (Seattle, WA, 10-14 abril, 2001). (ERIC Servicio de Reproducción de Documentos $\mathrm{N}^{\circ}$ ED452203).

Fernández, M. J., y González, A. (1997). Desarrollo y situación actual de los estudios de eficacia escolar. Revista Electrónica de Investigación y Evaluación Educativa, 3(1). Consultado el 10 de marzo del 2009, de http://www.uv.es/RELIEVE/v3n1/ RELIEVEv3n1_3.htm

Franco, S. M., y Tobasura, I. (2007). Familia, soberanía alimentaria y medio ambiente. Un caso de estudio. Revista Luna Azul, $\mathrm{N}^{\circ} 25$. Consultado el 30 de abril del 2009, de http://lunazul.ucaldas.edu.co/ index.php?option=com_content\&task $=$ view\&id=354\#ancla1

Gairín, J. (1990). Las actitudes en educación. Un estudio sobre educación matemática. Barcelona, España: Editorial Boixareu Universitaria.

García, F., y Doménech, F. (1997). Motivación, aprendizaje y rendimiento escolar. Revista Electrónica de Motivación y Emoción, 1(0). Consultado el 14 de setiembre del 2005, de: http://reme. uji.es/articulos/pa0001/texto.html

González, P. (1989). Aplicación del LISREL al análisis del rendimiento estudiantil. Revista Economía, $\mathrm{N}^{\circ} 4$, 55-73. Consultado el 10 de noviembre del 2004, de: http://iies.faces. ula.ve/Revista/Articulos/Revista_04/ rev4gonzalez.htm

Gutiérrez, L. (1994). La matemática escolarizada: ¿La ciencia transformada en dogma? Un estudio etnográfico realizado en aulas universitarias. Tesis doctoral sin publicar, Universidad Nacional Experimental Simón Rodríguez, Caracas, Venezuela.

Hwang, D. Y. (2002). A hierarchical linear modeling approach to higher education research: The influences of student and institutional characteristics [Un enfoque del modelo lineal jerárquico en la investigación de la educación superior: Las influencias de las caracteristicas institucionales $y$ del estudiante]. Artículo presentado en la Reunión Anual de la Asociación Suroeste de Investigación Educativa (Austin, TX, 14-16 febrero, 2002). (ERIC Servicio de Reproducción de Documentos $N^{\circ}$ ED466778).

Kerlinger, F. (1988). Investigación del comportamiento (3a. ed.). México: McGraw-Hill.

Kreft, I. G. (1990). Using multilevel analysis to assess school effectiveness: A study of Dutch secondary schools [Uso del análisis multinivel para evaluar la eficacia escolar: Un estudio de secundarias holandesas]. Artículo presentado en la Reunión Anual de la Asociación Americana de Investigación Educativa (San Francisco, CA, 27-31 marzo, 1989). (ERIC Servicio de Reproducción de Documentos $\mathrm{N}^{\circ}$ ED340721).

Malin, A., y Linnakyla, P. (2001). Multilevel modelling in repeated measures of the quality of Finnish school life [Modelo multinivel en medidas repetidas de la calidad de vida de escuela filandesa]. (ERIC Servicio de Reproducción de Documentos N EJ636333).

Martínez, V. (1996). Factores determinantes del rendimiento académico en 
enseñanza media. Revista Psicología Educativa, 2(1), 79-90.

Martínez, V. (2002). Condicionantes del rendimiento escolar. Educadores Revista de Renovación Pedagógica, $\mathrm{N}^{\circ} 204,285-295$.

Matas, A. (2003). Estudio diferencial de indicadores de rendimiento en pruebas objetivas. Revista Electrónica de Investigación y Evaluación Educativa, 9(2). Consultado el 05 de enero del 2005, de: http://www.uv.es/RELIEVE/v9n2/RELIEVEv9n2_5.htm

Mella, O., y Ortiz, I. (1999). Rendimiento escolar. Influencias diferenciales de factores externos e internos. $R e$ vista Latinoamericana de Estudios Educativos, 29(1), 69-92. Consultado el 16 de octubre del 2003, de: http://redalyc.uaemex.mx/redalyc/ pdf/270/27029103.pdf

Murillo, F. J. (1999). Los modelos jerárquicos lineales aplicados a la Investigación sobre eficacia escolar. Revista de Investigación Educativa, 17(2), 453-460.

Nunnally, J. C., y Bernstein, I. J. (1995). Teoría psicométrica. México: McGraw-Hill.

Ordoñez, Z. (2006). Estudio exploratorio sobre las prácticas de enseñanza adecuadas de los/las docentes. Una mirada a partir de los/las estudiantes [Versión electrónica]. Revista de Investigación, 6(2), 271-279. Recuperado el 6 de mayo del 2009, de http://redalyc.uaemex.mx/redalyc/ pdf/952/95260214.pdf

Ortega, J. A. (2001). Revisión de conceptos demográficos. Contribuciones a la Economía. Universidad de Málaga, España. Consultado el 16 de agosto del 2005, de: http://www.eumed.net/ cursecon/colaboraciones/Ortegademograf.htm

Pardo, A., y Ruiz, M. A. (2002). SPSS 11. Guía para el análisis de datos. España: McGraw-Hill.

Paterson, L. (1998). Multilevel multivariate regression: An illustration concerning school teachers' perceptions of their pupils [Regresión multivariada multinivel: Un ejemplo concerniente a las percepciones de los docentes acerca de sus estudiantes]. Investigación patrocinada por Grant R000234933 from the United Kingdom Economic and Social Research Council. (ERIC Servicio de Reproducción de Documentos $\mathrm{N}^{\circ}$ EJ572875).

Pong, S.-L. (1997). Estructura familiar, contexto escolar y el rendimiento de los alumnos del octavo grado en matemática y lectura. Journal of Marriage and the Family, 59(3), 734746. Resumen del artículo en inglés Family structure, school context, and eight grade math and reading achievement. Consultado el 26 de setiembre del 2005, de: http://www.grade. org.pe/IME/resumensp013.htm

Reimers, F. (2003). La buena enseñanza y el éxito escolar de los estudiantes en América Latina. Revista Iberoamericana de Educación, $\mathrm{N}^{\circ}$ 31. Consultado el 16 de febrero de 2005, de: http:// www.rieoei.org/rie31a01.PDF

Rojas, L. (2004). Factores asociados a la repitencia de los estudiantes que cursan sétimo año en colegios diurnos académicos públicos: Un análisis de niveles múltiples. Tesis doctoral sin publicar, Doctorado Latinoamericano en Educación, Universidad Estatal a Distancia, Costa Rica. 
Sainz, L. (1998). La enseñanza tutelar como modelo de comunicación pedagógica democrática. Revista Cubana Médica de Educación Superior, 12(2), 49-53. Consultado el 4 de abril del 2009, de: http://www.bvs.sld.cu/revistas/ems/ vol12_2_98/ems03298.pdf

Vélez, E., Schiefelbein, E., y Valenzuela, J. (1993). Factores que afectan el rendimientoacadémico enla educación primaria. Revista Iberoamericana de Educación. Consultado el 10 de febrero de 2005, de: http://www.oei. es/calidad2/Velezd.PDF

Young, D. J. (1997). A multilevel analysis of science and mathematics achievement [Unanálisismultiniveldellogroen matemáticas y ciencias]. Curtin University of Technology, Perth, Australia; Australian Research Council. Artículo presentado en la Reunión Anual de la Asociación Americana de Investigación Educativa (Chicago, IL, 24-28 marzo, 1997). (ERIC Servicio de Reproducción de Documentos N ED410242). 